Jingfeng $\mathrm{Hu}$

Yihan Zhang

Ping Wang

Fei Qin

http://dx.doi.org/10.21278/brod71206

\title{
NUMERICAL AND EXPERIMENTAL STUDY ON RESISTANCE OF ASYMMETRIC CATAMARAN WITH DIFFERENT LAYOUTS
}

\author{
UDC 629.5.015.2:629.5.018.71:629.5.022.22
}

Original scientific paper

\begin{abstract}
Summary
It is meaningful to study the wave-resistance reduction of an asymmetric catamaran because of interference effects between the two sets of ship-generated waves. The influence of lateral separation and longitudinal stagger on the resistance and the wave interference are analyzed within this paper. Numerical calculations of resistance, sinkage and trim of the asymmetric catamaran are carried out for several Froude number ranging from 0.24 to 0.48 , for six different lateral separations and four longitudinal staggers. Verification of numerical results is provided. The model tests are then carried out for three stagger distances to validate the numerical results. Results of this study indicate that the wave-resistance can be effectively reduced by certain hull layouts at different Froude numbers.
\end{abstract}

Key words: Asymmetric Catamaran; Resistance; Wave interference; CFD

\section{Introduction}

With the advent of the world's first asymmetric platform, a twin-hull private yacht "Asean Lady", the asymmetric catamaran has gradually attracted the attention of the public. Asymmetric catamarans usually consist of two hulls of different sizes and displacement, which can be propelled by pump jet, POD propeller and other vector propulsion methods because of yawing moment caused by asymmetric hull form. Asymmetric catamaran is not as prone to form a "spiral" swaying motion in waves like conventional catamaran, therefore its seakeeping is much better than conventional catamaran and has a good development prospect (Zhang et al., 2009[1]). In addition, the asymmetric catamaran not only has the advantages of spacious deck and good stability, but also has good resistance performance, which is not only due to its slender main hull, but also the favourable wave-making interference.

It should be noted that there are two forms of asymmetric catamaran generally. Typically, the asymmetric catamaran is consisted of two demi-hulls, which actually is a symmetrical form as a whole, such as those investigated by Mortaza et al. (2016)[2], Hajiabadi et al. (2017)[3], Yaakob et al. (2012)[4], Ikezoea et al. (2012)[5] and Kim et al. (2012)[6]. However, two different-size hulls with certain longitudinal and lateral distances 
from each other (termed as "stagger" and "separation") constitute an asymmetric catamaran has been rarely addressed. Li et al. (2013)[7] applied a time-domain panel method based on Green's theorem to solve the wave making problem of asymmetric catamaran, the wave making resistance of the asymmetric catamaran with different layouts was calculated, the analysis of interference effects varying with Froude number was also performed. Following that, Li et al. (2013)[8] utilized the hybrid genetic algorithm to optimize the configuration (transverse and longitudinal location of side hull) with minimum wave resistance. It was concluded that the favourable wave-making interference could be obtained by changing the relative position of side hull, in order to reduce resistance and improve speed. Yu et al. (2017)[9] studied the far-field wave interference and the resulting wave-resistance reduction of an asymmetric di-hull system, consisting of distinct Series 60 hull models with various hull configuration (various staggers and separations) by means of thin-ship computation and experiment. Obtained results showed that a considerable amount of wave-resistance reduction was indeed possible of the investigated hull configuration. However, beyond the far-field interference of the waves, near-field interactions of the two hulls were found to have nonnegligible influence on the total resistance performance of the di-hull system.

Although there is little literal research on the asymmetric catamarans, a large number of theoretical, experimental and numerical studies were performed on catamaran and trimaran, which have some similar characteristics with asymmetric catamarans. Many of these works were focused on the analysis of wave interference or configuration optimization. Insel and Molland (1992)[10] summarised an experimental and theoretical investigation into the interference effects on both wave resistance and viscous resistance for high speed displacement catamarans. Broglia et al. (2011)[11] carried out numerical simulations of viscous flow around catamaran and monohull models, which were fixed at the dynamic positions taken from the experiments. It was shown that dependence between interference effects and $\operatorname{Re}$ (Reynolds number) is weak. In order to study dependence between interference effects and separation, Zaghi et al. (2011)[12] performed extensive experiments and numerical simulations. Their investigations indicated that interference, as well as maximum resistance coefficient, is higher for catamaran configurations with smaller separation. The maximum value for interference increase and occurs at higher speeds as the separation decreases. Broglia et al. (2014)[13] also carried out experimental investigations for a highspeed catamaran. Results showed that the interference effects were more significant for narrower catamaran configurations and at intermediate values of $F n$. At larger hull spacing, the effect is attenuated and occurs at smaller $F n$. Authors also found that interference effects were strongly connected with sinkage and trim of the catamaran. Farkas et al. (2017)[14] investigated the influence of $F n$ and separations on both viscous interference and wave interference utilizing CFD. Since the viscous interference factor was found to be dependent on the separation within their studies, they claimed that viscous CFD methods should be used for interference investigation, rather than potential CFD methods. He et al. (2015)[15] demonstrated the applicability of URANS (Unsteady Reynolds Averaged Navier-Stokes) solver for the catamaran interference problems including the effects of sinkage and trim. They concluded that the main cause of the deviations in resistance, sinkage and trim using URANS solver was caused by the grid quality, but these numerical errors were found acceptable.

Besides catamaran, trimaran is also a mature ship type which has been widely studied and applied. The characteristics of resistance and roll motion of trimaran with variable layouts (different longitudinal and transverse position of side hull) were studied by means of both experiment and theoretical approaches in Cai et al. (2007)[16] and Wang et al. (2007)[17], where authors concluded that the aft longitudinal location of the side hull was better for high $F n$ trimaran, while the middle longitudinal location was better for small $F n$. Moreover, the transverse position of the side hull should be optimized to provide enough stability for the 
trimaran meanwhile the ship natural period should avoid the wave period. As was found by Mizine et al. (2009)[18], the hydrodynamic research and model testing of the large Trimaran Ship-Heavy Air Lift Ship (HALSS) showed a large change in resistance (70\%) due to a moderate (15\% from length of main hull) shift in the longitudinal side hull position, then several CFD calculations and tests were performed to analyse the influence of transverse and longitudinal location of side hull.

Nowadays, RANS based CFD methods were applied with success for the free surface simulations around ship, and widely used for optimization of the hull form. Duy et al. (2017)[19] used CFD for the optimal design for a stern shape of a hull. Muscari et al. (2017)[20] investigated hull-propeller-rudder interactions phenomena for twin-screw ships. Ozdemir et al. (2016)[21], Ozdemir et al. (2018)[22] and Farkas et al. (2017)[23] have investigated the free surface flow and total drag around ship hulls.

The resistance and the interference effect of asymmetric catamarans have not been fully studied. Moreover, many of the CFD simulations on the resistance of catamaran and trimaran vessels were performed for fixed models, or at the trim and sinkage taken from the experimental measurements (such as Broglia et al., 2011[11]; Zaghi et al. 2010[12]; Farkas et al. 2017[14] and Mizine et al. 2009[18]). As was concluded by Wang et al. (2011)[24], the precision of the trimaran wave resistance calculation was further improved by considering the trim and sinking, especially at high speeds. In this paper, the numerical simulations which consider sinkage and trim are carried out by using the commercial software package Star$\mathrm{CCM}+$. This work is focused on the analysis of the resistance characteristics and wave interference of an asymmetric catamaran with varying the transverse and longitudinal locations of side hull. In order to gain a better insight into the interference phenomena and the flow field around the asymmetric catamaran, the analysis, in terms of wave patterns, wave cuts at two values of Froude number $(F n=0.29,0.40)$ is performed. Moreover, the experiments are carried out for three longitudinal distances at the Model towing tank of Harbin Engineering University. Numerical results of resistance, trim and sinkage are compared to the experimental measurements. The research results of this paper can provide some useful reference for the design of asymmetric catamarans.

\section{Geometry}

The research object of this paper is a 2500 tons rescue vessel in design stage, of which the main dimensions and particulars in the model scale are given in Table 1. The hull lines of the asymmetric catamaran are shown in Fig. 1.

Table 1 The main dimensions and particulars of the asymmetric catamaran model

\begin{tabular}{|c|c|c|c|c|c|}
\hline Parameters items & \multicolumn{2}{|c|}{ Main hull parameters } & Side hull parameters & Unit \\
\hline Waterline length & $L_{m}$ & 3 & $L_{s}$ & 1.429 & $m$ \\
\hline Draft & $d_{m}$ & 0.120 & $d_{s}$ & 0.074 & $m$ \\
\hline Waterline width & $B_{m}$ & 0.286 & $B_{s}$ & 0.071 & $m$ \\
\hline Displacement & $\Delta_{m}$ & 47.158 & $\Delta_{s}$ & 3.475 & $\mathrm{~kg}$ \\
\hline Wetted surface & $S_{m}$ & 0.990 & $S_{s}$ & 0.210 & $\mathrm{~m}^{2}$ \\
\hline Block coefficient & $C_{B m}$ & 0.445 & $C_{B s}$ & 0.445 & \\
\hline Scale ratio & & 36.5 & & 36.5 & \\
\hline
\end{tabular}


Hu Jingfeng, Zhang Yihan, Wang Ping, Qin Fei
Numerical and Experimental Study on Resistance of Asymmetric Catamaran with Different Layouts

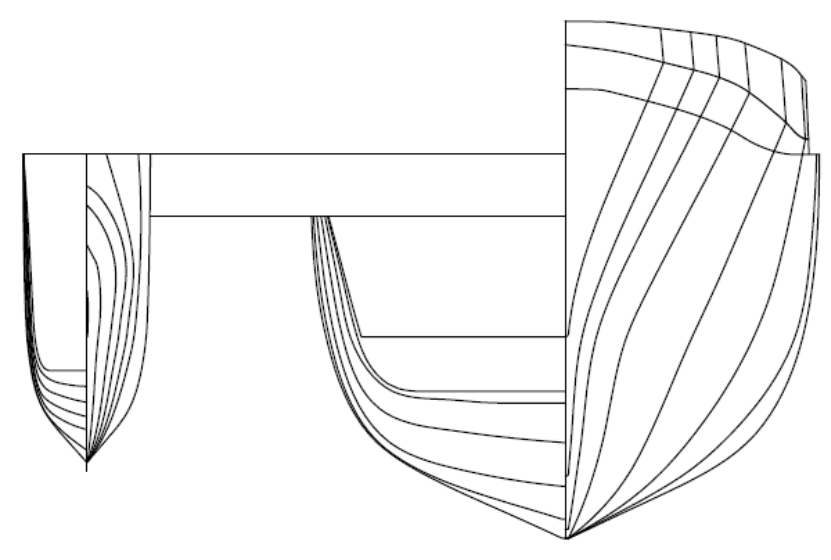

Fig. 1 The hull lines of the asymmetric catamaran

The relative centerline distance between the main and side hull is defined as $C L$, the relative stagger distance between the main and side hull is defined as $S T$.

$$
\begin{aligned}
& C L=\frac{C}{B_{\mathrm{m}}} \\
& S T=\frac{S}{L_{\mathrm{m}}}
\end{aligned}
$$

Where: $C$ is the centerline distance between the main and side hull, $S$ is the stagger distance between the main and side hull, the geometric arrangement of the asymmetric catamaran is shown in Fig. 2. The different layouts studied in this paper are shown in the Table 2 and Table 3, which are six different lateral separation layouts and four different longitudinal stagger layouts.

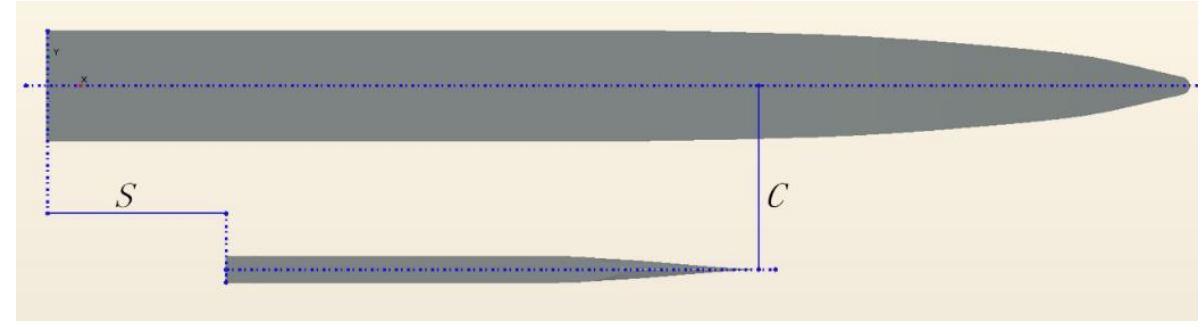

Fig. 2 The geometric arrangement of asymmetric catamaran

Table 2 Different lateral separation layouts of the asymmetric catamaran

\begin{tabular}{|c|c|c|c|c|c|c|}
\hline Scheme code & S1C1 & S1C2 & S1C3 & S1C4 & S1C5 & S1C6 \\
\hline$C L$ & 0.8 & 1.0 & 1.2 & 1.4 & 1.76 & 2.0 \\
\hline$S T$ & 0 & 0 & 0 & 0 & 0 & 0 \\
\hline
\end{tabular}

Table 3 Different longitudinal stagger layouts of the asymmetric catamaran

\begin{tabular}{|c|c|c|c|c|}
\hline Scheme code & S1C5 & S2C5 & S3C5 & S4C5 \\
\hline$C L$ & 1.76 & 1.76 & 1.76 & 1.76 \\
\hline$S T$ & 0 & 0.062 & 0.162 & 0.25 \\
\hline
\end{tabular}




\section{Numerical calculation method}

\subsection{Numerical setup}

Overset mesh is adopted in this work, which is much useful in dealing with large motions of high-speed ship (ZHAO et al., 2011[25]). Thus the calculation domain is divided into an overlap domain and a background domain, as shown in Fig. 3. The boundary conditions of the background domain and the domain dimension are shown in Table 4. The overlap domain is a rectangular enclosing the hull, its length, width and height are $3.2 \mathrm{Lm}$, $3.5 \mathrm{Bm}$ and $5 \mathrm{dm}$, respectively. Therefore, the asymmetric catamaran for the largest separation ratios can also be enclosed. The grid near the free surface and the hull are refined gradually, and the area encapsulating Kelvin wave is also refined. The grid around the hull is shown in Fig. 4.

The DFBI (Dynamic Fluid Body Interaction) model is used to simulates the motion of the ship according to the forces acting on it induced by the flow. For this simulation, 2 degrees of freedom to account for sinkage and trim is allowed. The VOF model is applied to trace the location and evolution of free surface. And the VOF wave damping is applied to inlet, outlet and side boundary. Within STAR-CCM+, this wave damping approach is implemented refer to Choi and Yoon (2009)[26].

The implicit unsteady solver is chosen for the unsteady calculations. A cell based FVM is used for discretization of the governing equations. Convection terms in RANSE are discretized with a second order upwind scheme, while temporal is discretized with first order upwind scheme. The discretized formulations are solved by using a Rhie-and-Chow-type pressure-velocity coupling combined with a SIMPLE algorithm. The simulations are stopped when the values of total resistance became steady, i.e. when the oscillation amplitude near the converged value of total resistance fell below $0.5 \%$.

Table 4 Boundary condition and dimension

\begin{tabular}{|c|l|l|}
\hline Boundary & \multicolumn{1}{|c|}{ Condition } & \multicolumn{1}{c|}{ Domain dimension } \\
\hline Inlet & Velocity inlet condition & $1.5 L_{m}$ from the bow \\
\hline Outlet & Pressure outlet & $3 L_{m}$ from the stern \\
\hline Top & Velocity inlet condition & $1.5 L_{m}$ from the waterline \\
\hline Bottom & Velocity inlet condition & $2 L_{m}$ from the waterline \\
\hline Side & Velocity inlet condition & $\begin{array}{l}2 L_{m} \text { from the longitudinal section of the } \\
\text { main hull }\end{array}$ \\
\hline
\end{tabular}


Hu Jingfeng, Zhang Yihan, Wang Ping, Qin Fei
Numerical and Experimental Study on Resistance of Asymmetric Catamaran with Different Layouts

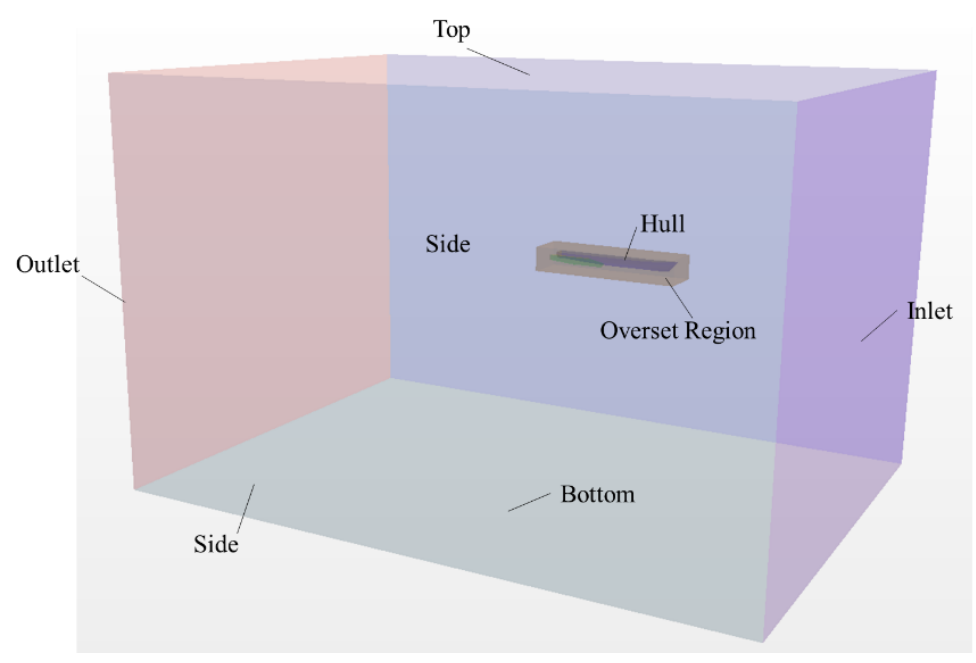

Fig. 3 Boundaries representation for overlap and background regions
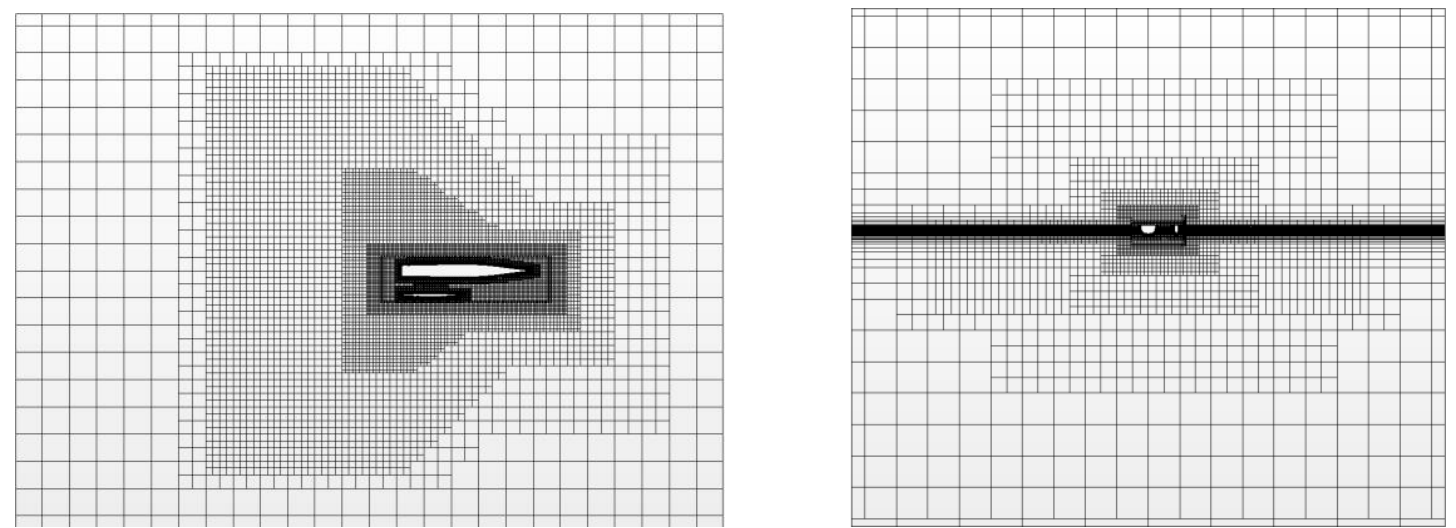

Fig. 4 Mesh visualization around the hull

In this paper, the $\mathrm{k}-\varepsilon$ turbulence model is adopted in the numerical calculations, which is only valid for fully developed turbulent core regions. Thus the standard wall function method is used to solve the flow in the near wall region. The wall function is a hybrid approach, as indicated by Mancini et al. (2018)[27], that attempts to emulate the high $y+$ wall treatment for coarse meshes (for $y+>30$ ), and the low $y+$ wall treatment for fine meshes (for $y+\approx 1$ ). This approach is formulated to assure reasonable answers for meshes of intermediate resolution considered as the best compromise between the describing of the boundary layer with acceptable quality and the time required for the simulation. According to the ITTCRecommended Procedures and Guidelines (2014)[28], 30 $\leq y+\leq 100$ is recommended for standard wall functions, where $y+$ is calculated by the following equations (3) and (4). In addition, as reported by DENG et al. (2013) [29], the resistances of a monohull calculated with four sets of grids with $y+$ of $11.5,60,120$, and 200 respectively, using the $\mathrm{k}-\varepsilon$ turbulence model, were almost the same.

$$
\begin{gathered}
\mathrm{y}=\frac{y^{+} L_{w l}}{R e \sqrt{C_{f} / 2}} \\
C_{f}=\frac{0.075}{\left(\log _{10}(R e)-2\right)^{2}}
\end{gathered}
$$


Where: $y+$ is the dimensionless coefficient of the first layer of grid height; $y$ is the height of the first layer of the boundary layer; $L_{w l}$ is the waterline length; $R e$ is the Reynolds number; $C_{f}$ is the frictional resistance coefficient.

In this paper, the height of the first layer grid is estimated for $F n$ value of 0.4 and $y+$ value of 60. Then a 5-layer prism layer grid is divided by a grid growth ratio of 1.2. Fig. 5 and Fig. 6 show the $y+$ values of the hull surface at the minimum ship velocity $(F n=0.24)$ and the maximum ship velocity $(F n=0.48)$, respectively. It can be seen that the $y+$ value distribution is basically within a reasonable range.

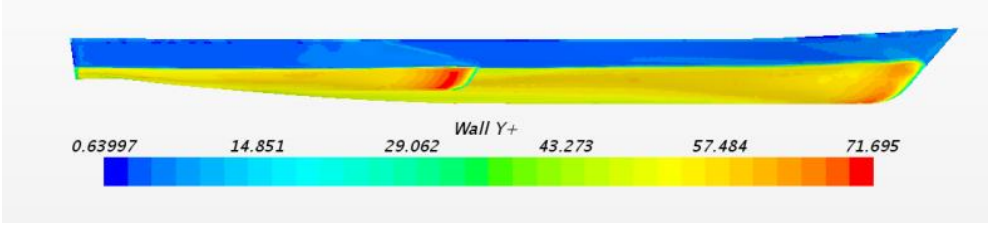

Fig. 5 Wall $y+$ visualization on the ship hull at $F n=0.24$

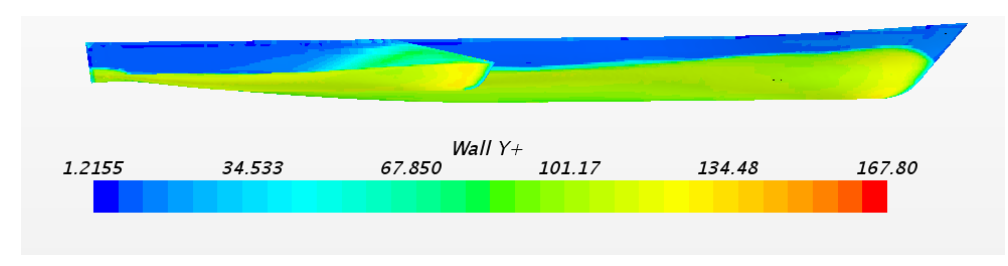

Fig. 6 Wall $y+$ visualization on the ship hull at $F n=0.48$

\subsection{Convergence study}

Convergence study has been carried out according to ITTC procedure (ITTC, 2002 ${ }^{[30]}$ ) for time step and mesh size. Changes between medium-fine $\varepsilon_{k, 21}=\hat{S}_{k, 2}-\hat{S}_{k, 1}$ and coarsemedium $\varepsilon_{k, 32}=\hat{S}_{k, 3}-\hat{S}_{k, 2}$ solutions are used to define the convergence ratio and to determine convergence condition. The convergence ratio is defined as follows:

$$
R_{k}=\frac{\varepsilon_{k, 21}}{\varepsilon_{k, 32}}
$$

where $\hat{S}_{k, 1}, \hat{S}_{k, 2}$ and $\hat{S}_{k, 3}$ correspond to solutions with fine, medium, and coarse input parameter, respectively. Three convergence conditions are possible:

(i) Monotonic convergence: $0<R_{k}<1$

(ii) Oscillatory convergence: $R_{k}<0$

(iii) Divergence: $R_{k}>1$

For monotonic convergence condition, generalized Richardson extrapolation (RE) is used to estimate uncertainties $U_{k}$ and errors $\delta_{R E}$. RE error is computed according to:

$$
\delta_{R E}=\frac{\varepsilon_{k, 21}}{r_{k}^{P_{k}}-1}
$$

where $r_{k}$ stands for uniform refinement ratio. $P_{k}$ is the order of accuracy calculated with following equation:

$$
P_{k}=\frac{\operatorname{In}\left(\varepsilon_{k, 32} / \varepsilon_{k, 21}\right)}{\operatorname{In}\left(r_{k}\right)}
$$


The uncertainty for monotonic convergence is calculated according to safety factor method as follow:

$$
U_{k}=\frac{F_{S}\left|\delta_{R E}\right|}{\left|\hat{S}_{k, 1}\right|}
$$

where $F S$ is the safety factor and is recommended to be equal to 1.25. (Farkas et al., (2017) [14]; ITTC, 2002[30]; Yengejeh et al., 2016[31])

The verification procedure is performed for the configuration S1C5 for Fn $=0.4$. Firstly, verification for time step is made using medium mesh. Three time steps are chosen with the ratio of 2, namely $0.04 s, 0.02 s$ and $0.01 s$. The obtained results are shown in Table 5. The convergence study for mesh size is carried out for medium time step $0.02 \mathrm{~s}$. Three grid cases, i.e. coarse mesh of around 0.72 million cells, medium mesh of around 1.43 million cells, and fine mesh of around 2.71 million cells, are obtained with refinement ratio equal to $\sqrt{2}$, while the boundary layer grid size keeps unchanged. The obtained results are show in Table 6.

In general, very low uncertainties are estimated for resistance and sinkage, while the obtained uncertainty for trim are larger. The medium time step $(0.02 s)$ and medium mesh (1.43 million cells) can be a good compromise between accuracy and computational time, thus they were utilized in the numerical calculations.

Table 5 Verification of time step for S1C5 for $F n=0.4\left(R_{t}\right.$ is the total resistance, Si is the sinkage)

\begin{tabular}{|c|c|c|c|c|c|c|c|c|c|}
\hline Items & $\hat{S}_{k, 3}$ & $\hat{S}_{k, 2}$ & $\hat{S}_{k, 1}$ & $\varepsilon_{k, 21}$ & $\varepsilon_{k, 32}$ & $R_{k}$ & $P_{k}$ & $\delta_{R E}$ & $U_{k}, \%$ \\
\hline$R_{t}(\mathrm{~N})$ & 14.632 & 14.668 & 14.697 & -0.029 & -0.036 & 0.806 & 0.624 & -0.054 & 0.456 \\
\hline $\operatorname{Si}(m m)$ & -6.708 & -6.859 & -6.950 & 0.091 & 0.151 & 0.603 & 1.461 & 0.052 & 0.933 \\
\hline $\operatorname{Trim}\left(^{\circ}\right)$ & -0.085 & -0.096 & -0.102 & 0.006 & 0.011 & 0.545 & 1.749 & 0.003 & 3.114 \\
\hline
\end{tabular}

Table 6 Verification of mesh size for S1C5 for $F n=0.4$

\begin{tabular}{|c|c|c|c|c|c|c|c|c|c|}
\hline Items & $\hat{S}_{k, 3}$ & $\hat{S}_{k, 2}$ & $\hat{S}_{k, 1}$ & $\varepsilon_{k, 21}$ & $\varepsilon_{k, 32}$ & $R_{k}$ & $P_{k}$ & $\delta_{R E}$ & $U_{k}, \%$ \\
\hline$R_{t}(\mathrm{~N})$ & 14.817 & 14.668 & 14.666 & 0.002 & 0.149 & 0.013 & 12.438 & 0.00003 & 0.0002 \\
\hline $\operatorname{Si}(m m)$ & -7.001 & -6.859 & -6.795 & -0.064 & -0.142 & 0.451 & 2.299 & -0.053 & 0.966 \\
\hline $\operatorname{Trim}\left({ }^{\circ}\right)$ & -0.108 & -0.096 & -0.089 & -0.007 & -0.012 & 0.583 & 1.555 & -0.010 & 13.76 \\
\hline
\end{tabular}

\section{Resistance characteristics}

\subsection{Analysis of numerical results of different lateral layouts}

This section is devoted to the study of the lateral location of side hull at the stagger distance of 0. Numerical simulations are carried out for two Froud numbers $(F n=0.29$ and $F n$ $=0.4$ ), for six separation ratios $(C L)$. The simulation results of total resistance are shown in the Table 6, and comparison of total resistance vary with different $C L$ is plotted in Fig. 7.

Table 6 Resistance of different lateral separation layouts (Unit: N)

\begin{tabular}{|c|c|c|c|c|c|c|}
\hline$F n$ & S1C1 & S1C2 & S1C3 & S1C4 & S1C5 & S1C6 \\
\hline 0.29 & 7.75 & 7.73 & 7.76 & 7.96 & 7.99 & 8.00 \\
\hline 0.4 & 15.29 & 15.15 & 14.73 & 14.43 & 14.67 & 14.5 \\
\hline
\end{tabular}




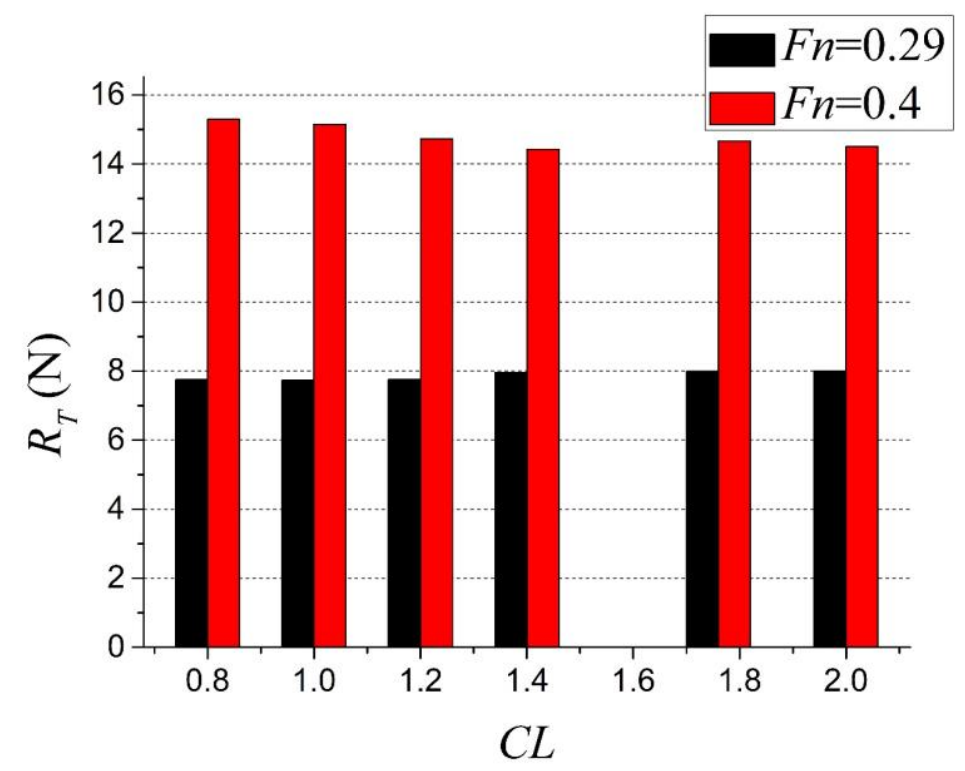

Fig. 7 Comparison of resistance between different lateral layouts

The obtained results show that the resistance change is very small at $F n=0.29$, less than $0.5 \%$. At $F n=0.4$, and separation distance is less than $1.4 B_{m}$, increasing lateral separation has a certain effect on reducing resistance, and maximum reduction is about $5 \%$. While changing separation distance has little effect on resistance when the separation distance is greater than $1.4 B_{m}$.

4.2. Analysis of numerical results of different longitudinal layouts

In this section, the asymmetric catamaran is investigated with a special focus on the influence of the longitudinal location of the side hulls. Four configurations (namely S1C5, S2C5, S3C5 and S4C5, which are listed in Table 3) are obtained by changing the longitudinal position of the side hull and keeping the separation distance of $1.76 B_{m}$ unchanged.

Comparing CFD friction resistance with the estimated friction resistance estimated by 1957 ITTC formula (4), the results are reported in Fig. 8. It can be observed that the change of spacing has little effect on friction resistance, and the CFD values are basically consistent the estimated values. The slight difference of the frictional resistance shows the viscous interference of main and side hulls. The viscous interference is mainly caused by occurrence of the cross flow and the changing of wetted surface (Farkas et al., 2017[14]). 


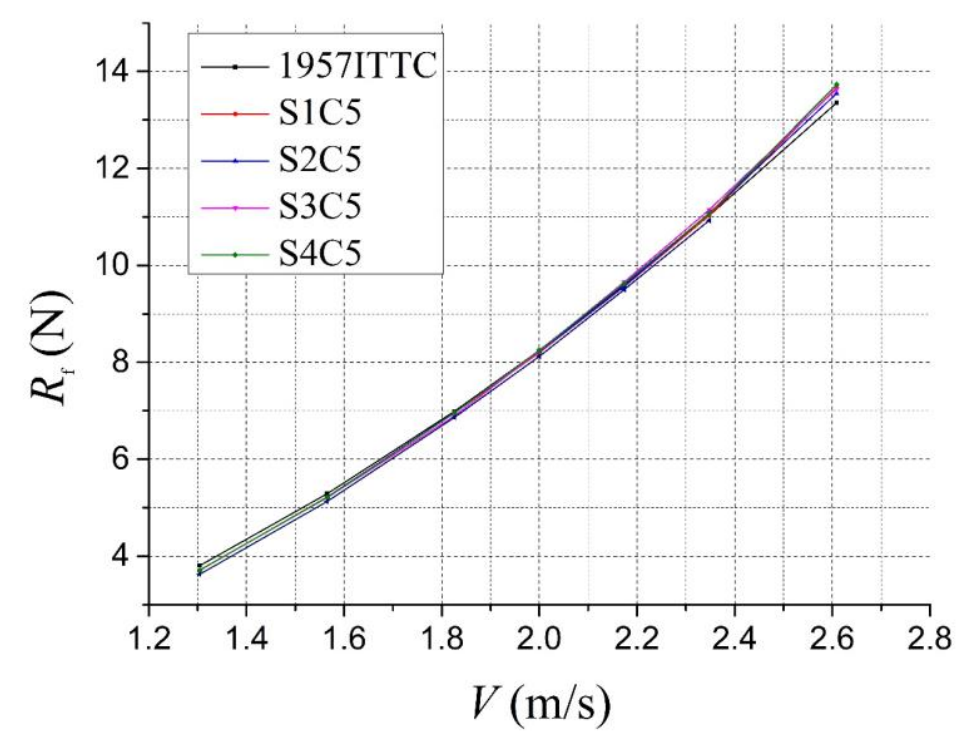

Fig. 8 Comparing CFD friction resistance with the estimated friction resistance

The frictional resistance coefficients of the side hull and the main hull are calculated according to the formula (4). The total frictional resistance is calculated as follows:

$$
R_{\mathrm{f}}=C_{\mathrm{f} m} \times S_{m} \times \frac{1}{2} \rho v^{2}+C_{\mathrm{f} s} \times S_{s} \times \frac{1}{2} \rho v^{2}
$$

Where: $C_{\mathrm{fm}}$ is friction resistance coefficient of main hull, $C_{\mathrm{fs}}$ is friction coefficient of side hull, $S_{\mathrm{m}}$ is wet surface area of main hull, $S_{S}$ is wet surface area of side hull, $\rho$ is the density of water, $v$ is the ship velocity.

In Fig. 9, the total resistance coefficient, residual resistance coefficient, sinkage and trim versus Froude number are shown for four configurations of difference stagger distances. The sinkage value in the figure refers to the vertical change of the ship's gravity center relative to the geodetic coordinate system, of which upward is positive. And when the trim is positive means the hull bow down. The total resistance coefficient and residual resistance coefficient are calculated with the following equations:

$$
\begin{aligned}
& C_{\mathrm{t}}=\frac{R_{T}}{\frac{1}{2} \rho v^{2}\left(S_{m}+S_{s}\right)} \\
& C_{\mathrm{r}}=\frac{R_{T}-R_{\mathrm{f}}}{\frac{1}{2} \rho v^{2}\left(S_{m}+S_{s}\right)}
\end{aligned}
$$

Where: $R_{T}$ is the total resistance, $R_{\mathrm{f}}$ is the residual resistance.

From Fig. 9, it can be seen that the total resistance coefficient and residual drag coefficient show almost the same rule. At low speed $(F n=0.24 \sim 0.35)$, the value of resistance increases as the decrease of stagger distance $(S L)$, and the drag increases by less than $5 \%$. While at higher speed $(F n=0.35 \sim 0.48)$, the shorter the stagger distance, the smaller are the drag, sinkage and trim. The maximum drag reduction, at $F n=0.43$, of layout S1C5 can reach $14 \%$ compared with that of layout S4C5. 

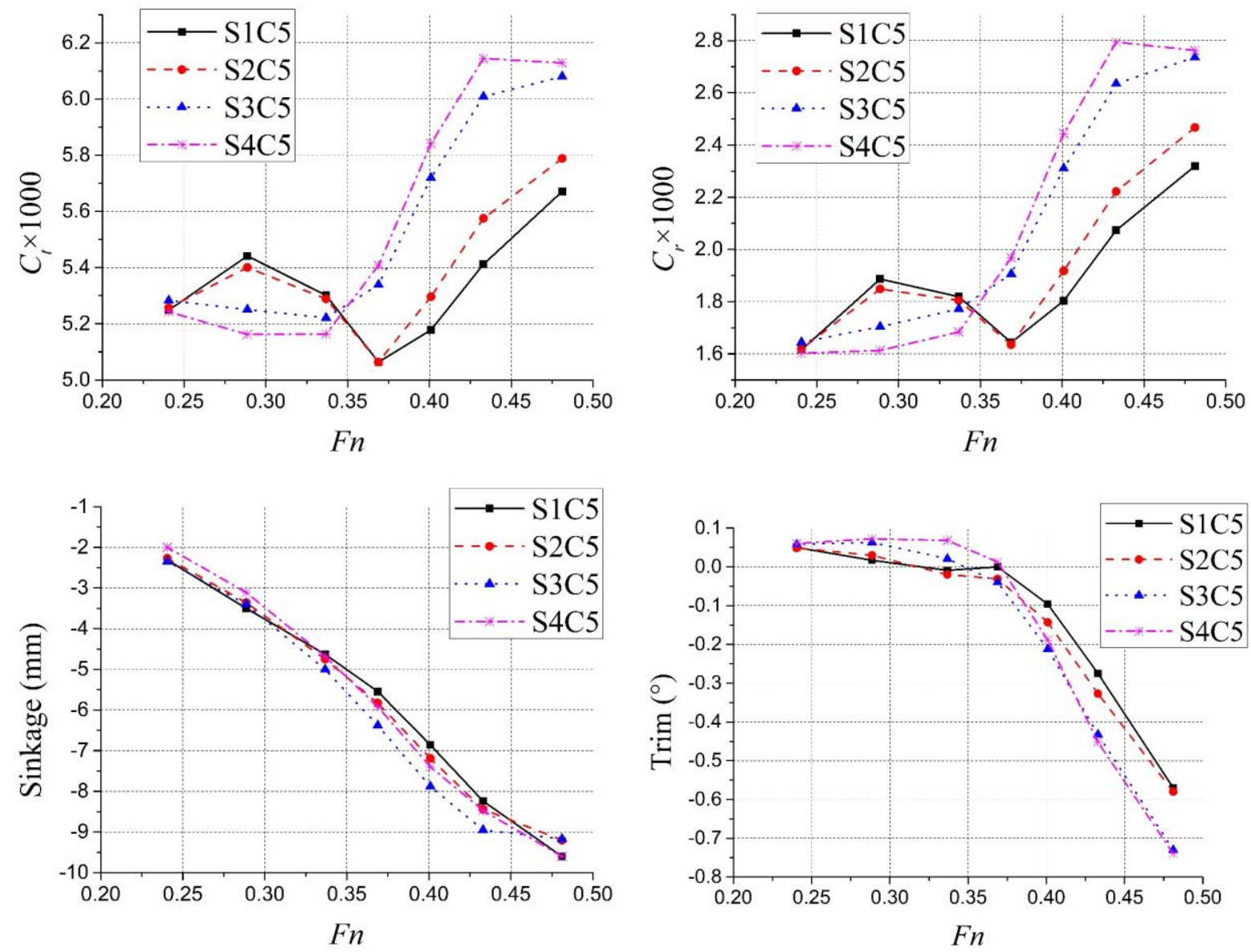

Fig. 9 CFD results vary with Froude number for different longitudinal layouts

In addition, it also can be seen from Fig. 9 that the resistance discrepancy between different staggering layouts is mainly due to the wave-making resistance. The wave-making resistance is proportional to the square sum of wave height and wave width. Therefore, when the wave-making height increases, the wave-making resistance will increase sharply. The wave length of transverse wave is about half of the main hull's Waterline length at $F n=0.29$, while it is approximately equal to the main hull's Waterline length at $F n=0.4$. As shown in Fig. 9, the resistance variation trend changing with the stagger distance is completely opposite at these two speeds. In order to gain a better insight into the interference phenomena, wave patterns and wave cuts are analyzed for these two speeds.

The position where three wave cuts are taken is shown in Fig. 10; the wave cut 1 is taken at the longitudinal axis of the main hull; the wave cut 2 is taken at a distance of Y2 = $0.3058 \mathrm{~m}$ from the longitudinal axis of the main hull, at the middle position of the inner region; the wave cut 3 is taken at the longitudinal axis of the side hull. 
Hu Jingfeng, Zhang Yihan, Wang Ping, Qin Fei
Numerical and Experimental Study on Resistance of Asymmetric Catamaran with Different Layouts

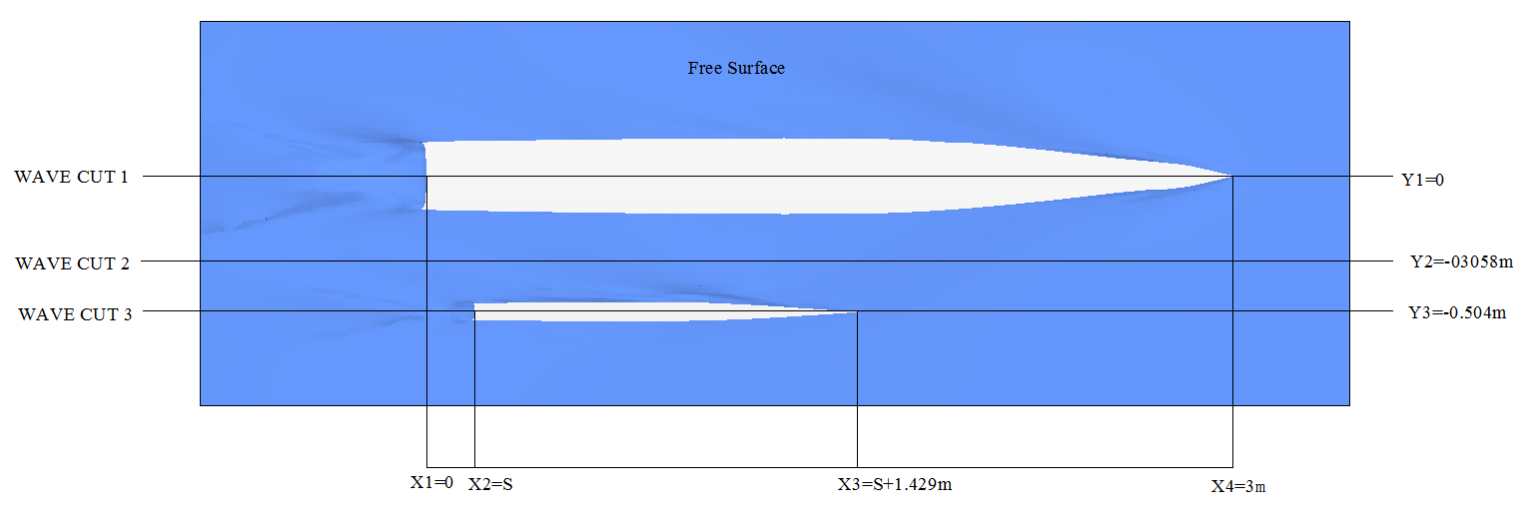

Fig. 10 Positions of wave cuts (Where, $Y 2=\frac{\left(C-\left(B_{s}+B_{m}\right) / 2\right)}{2}+B_{m} / 2, Y 3=C$ )
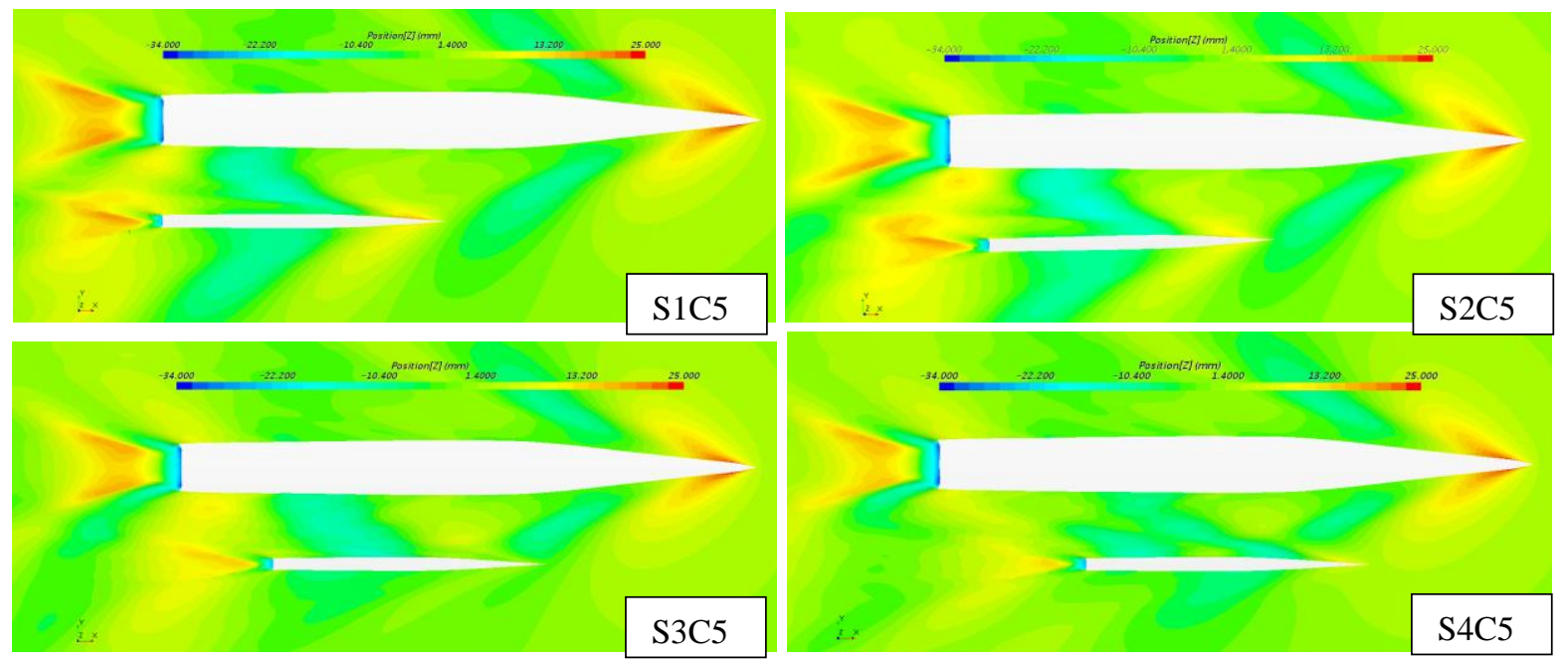

Fig. 11 Contours of wave pattern for different longitudinal layouts for $F n=0.29$

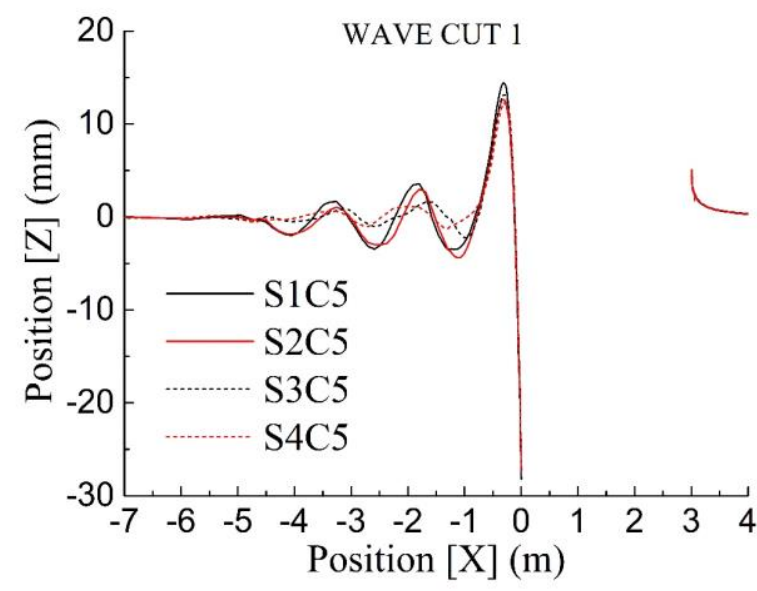

(a) Wave-cut profiles at $\mathrm{Y} 1$

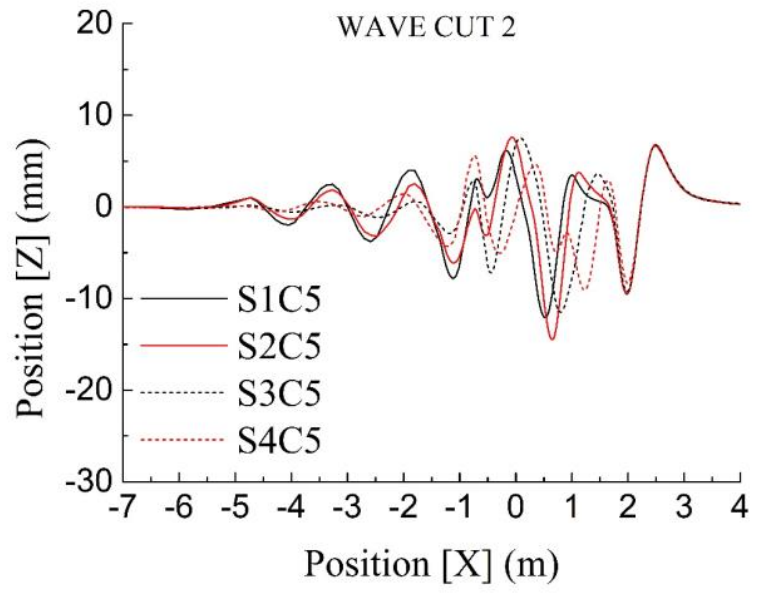

(b) Wave-cut profiles at Y2 


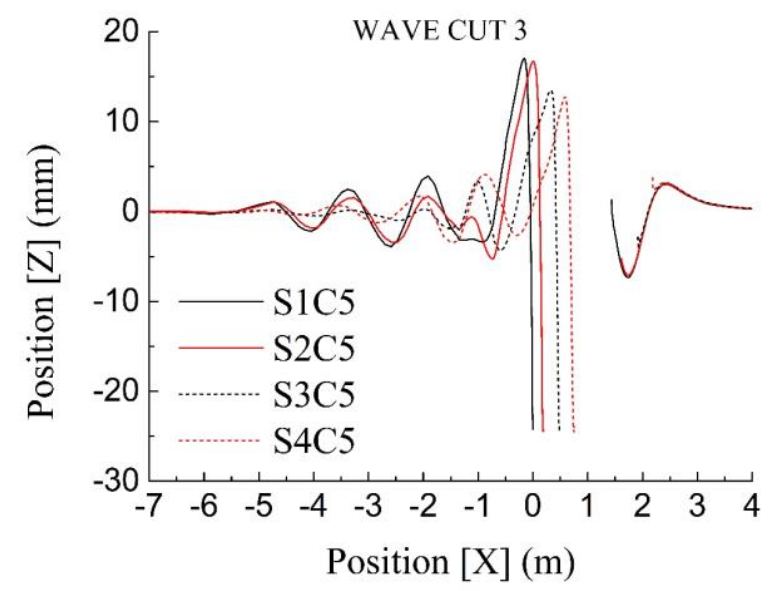

(c) Wave-cut profiles at $\mathrm{Y} 3$

Fig. 12 Wave-cut profiles for different longitudinal layouts for $F n=0.29$

The contours of wave pattern and wave-cut profiles at $F n=0.29$ are shown in Fig.11 and Fig.12, respectively. It can be seen that, at $F n=0.29$, the bow of the side hull is located in the wave trough behind the main hull for the layout S2C5 and S3C5, which is beneficial to reducing the bow wave amplitude of side hull. Moreover, the forward placing of the side hull can effectively reduce the stern wave-making. Consequently, the residual resistance is reduced.
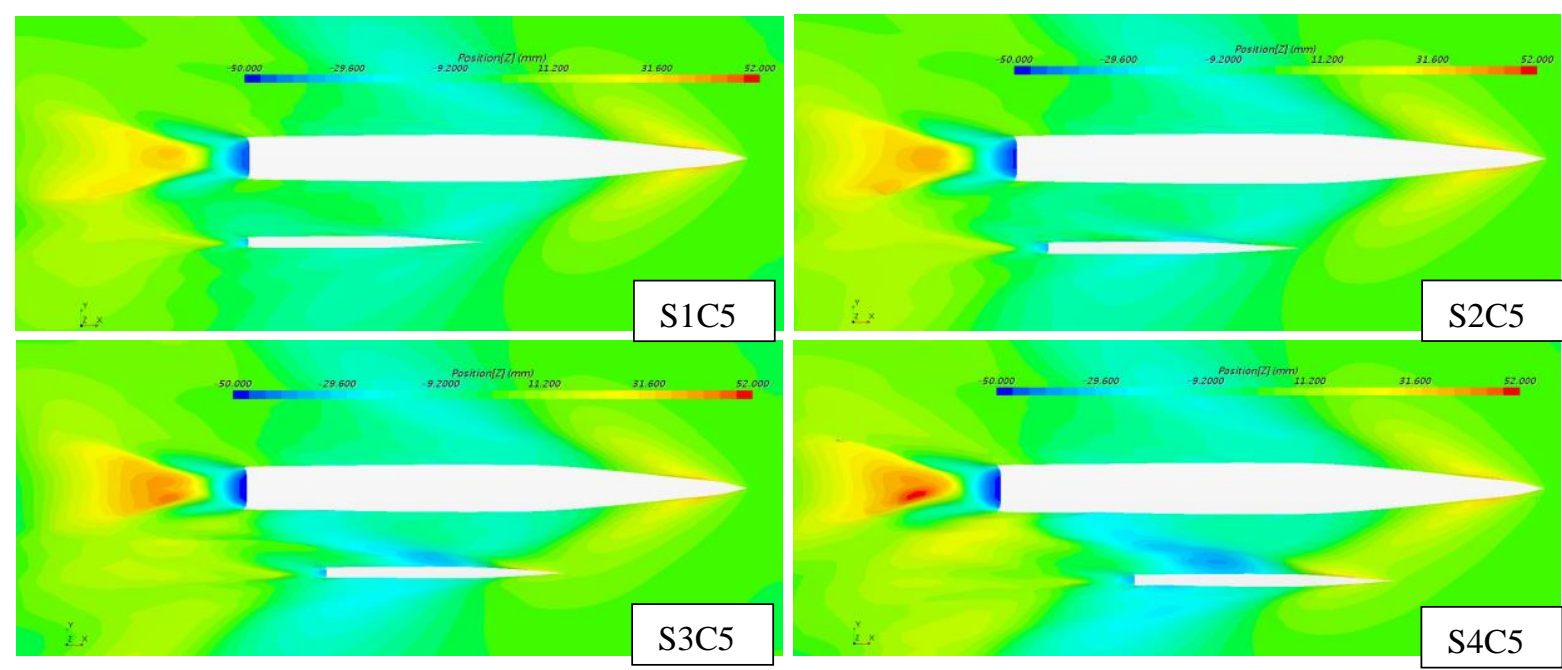

Fig. 13 Contours of wave pattern for different longitudinal layouts for $F n=0.4$ 
Hu Jingfeng, Zhang Yihan, Wang Ping, Qin Fei
Numerical and Experimental Study on Resistance of Asymmetric Catamaran with Different Layouts

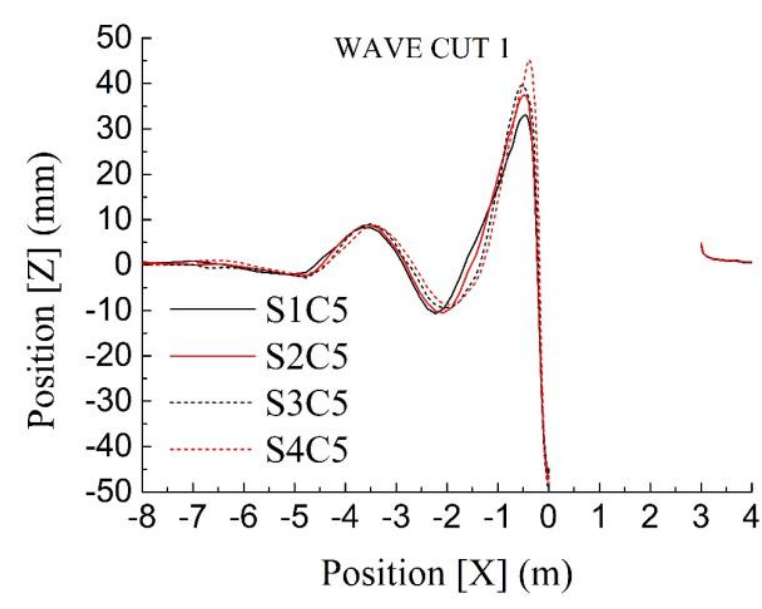

(a) Wave-cut profiles at $\mathrm{Y} 1$

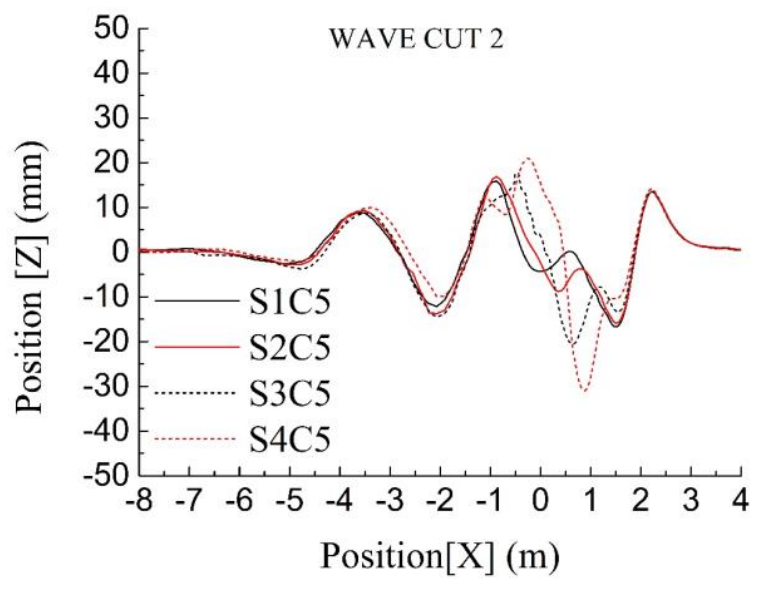

(b) Wave-cut profiles at Y2

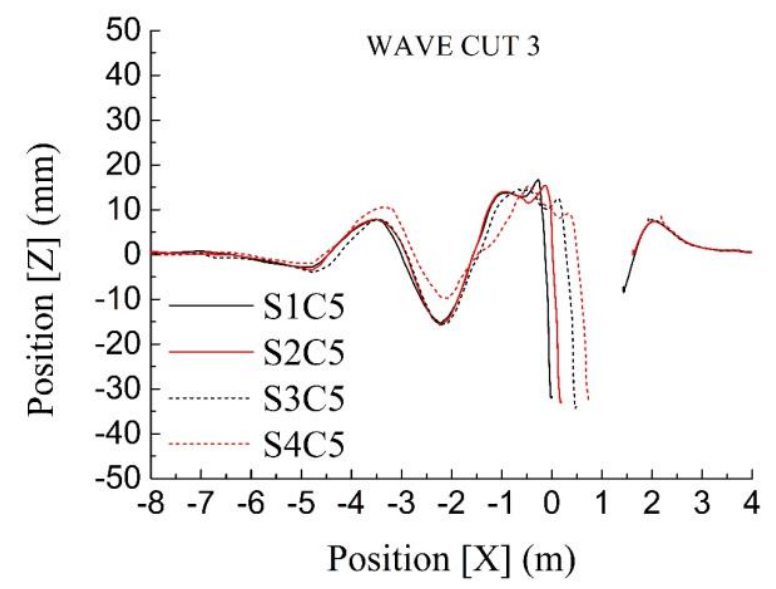

(c) Wave-cut profiles at $\mathrm{Y} 3$

Fig. 14 Wave-cut profiles for different longitudinal layouts for $F n=0.4$

The contours of wave pattern and wave-cut profiles at $F n=0.40$ are shown in Fig. 13 and Fig. 14, respectively. It can be seen that the wave-making amplitude near the inner side is obviously larger than that on the outer side of the side hull at $F n=0.4$. As the side hull being placed forward, a strong wave interaction between main and side hulls occurring. Particularly, one can notice a deep trough near the inner side of the side hull, also the stern wave height of the main hull increased, which may explain the higher residual resistance of S3C5 and S4C5. Moreover, when the side hull is near the middle of the main hull such as the layout S4C5, the adverse wave-making interference intensifies, and the inner part of the side hull forms obvious vortices, which will cause a significant increase in the resistance. While when the side hull is relatively backward, the bow of side hull is located in the trough of the wavemaking of main hull, which is beneficial to reducing the wave-making resistance of the side hull.

\subsection{Experimental validation}

The experiments reported here have been carried out at the Model towing tank of Harbin Engineering University, which is a $108 \mathrm{~m}$ long, $7 \mathrm{~m}$ wide and $3.5 \mathrm{~m}$ deep facility, as shown in the Fig. 16. As reported by ZHOU et al. (2006)[32], repeatability tests $(F n=0.147$, $0.220,0.300$ ) have been carried out on a $3 \mathrm{~m}$ FRP ship model in this tank. The uncertainty for total resistance is respectively equal to $1.451 \%, 0.675 \%, 0.295 \%$.

Attitude and resistance tests have been performed with the asymmetric catamaran models attached to the carriage of the tank in such a way that the models were free to heave 
and pitch while yaw was not permitted. In order to achieve the turbulent flow, the main hull of the model was equipped with turbulence stimulators. The tests have been conducted at seven values of Froude number $(F n=0.24,0.29,0.34,0.39,0.4,0.43$ and 0.48$)$ and for three stagger distances of the asymmetric catamaran model, namely S1C5, S2C5 and S3C5. The models were towed horizontally with a Four degrees of freedom airworthiness instrument (GEL-421-1, made in Japan) through the Longitudinal center of gravity (LCG, see Fig. 15), on the longitudinal axis of the main hull. Fig. 17 shows the test case of S1C5 at Fn=0.4.

The velocity of the towing carriage was measured by an encoder with uncertainty better than $1 \mathrm{~mm} / \mathrm{s}$ over the entire range of velocities. The sinkage and total resistance were measured by the Four degrees of freedom airworthiness instrument (the load cell is strain type, with a range of $10 \mathrm{~kg}$ and a measurement accuracy of $0.16 \%$; the measurement range of sinkage is $\pm 200 \mathrm{~mm}$, with a measurement accuracy of $0.1 \%$ ). The trim was measured by an Angle sensor (measurement range of $\pm 20^{\circ}$, measurement accuracy of $0.01 \%$ ), which located at the LCG, beside the load cell. Resistance time-series were collected at a sampling rate of $200 \mathrm{~Hz}$ with a 12-bit A/D board, and the acquired datas were time-averaged to provide mean values for each run.

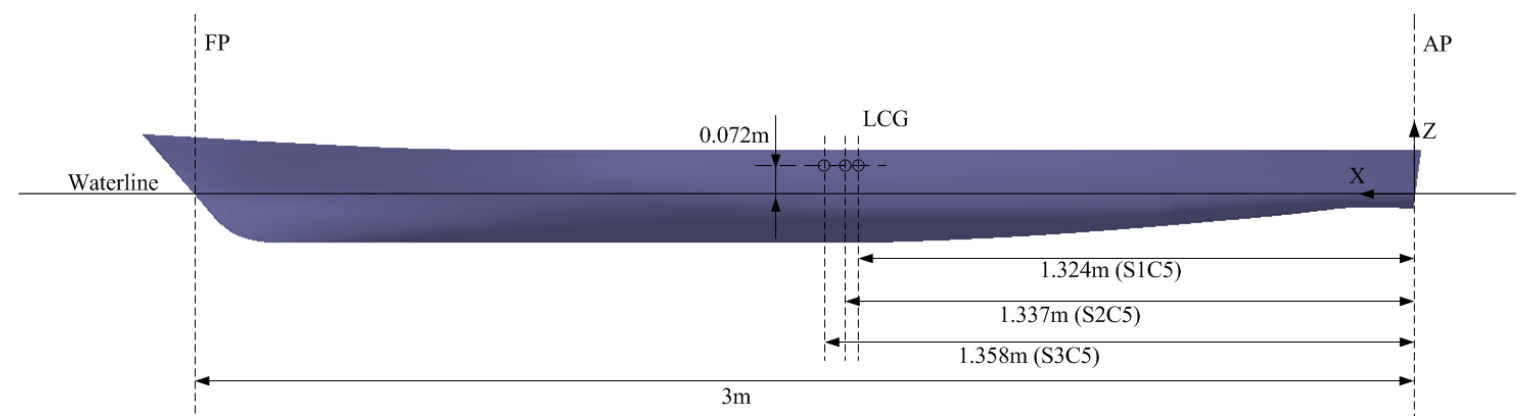

Fig. 15 The LCG of the asymmetric catamaran models

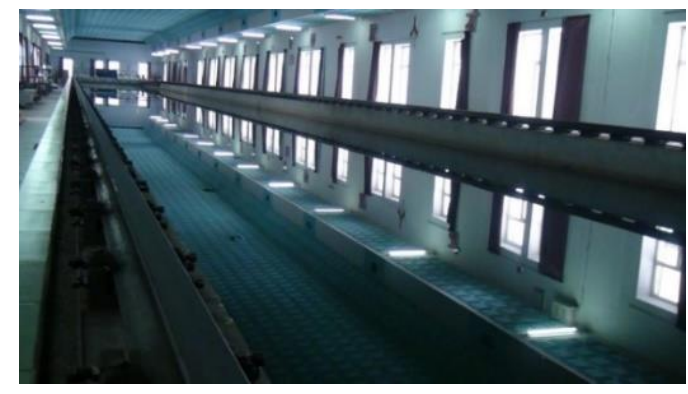

Fig. 16 Photograph of the model towing tank

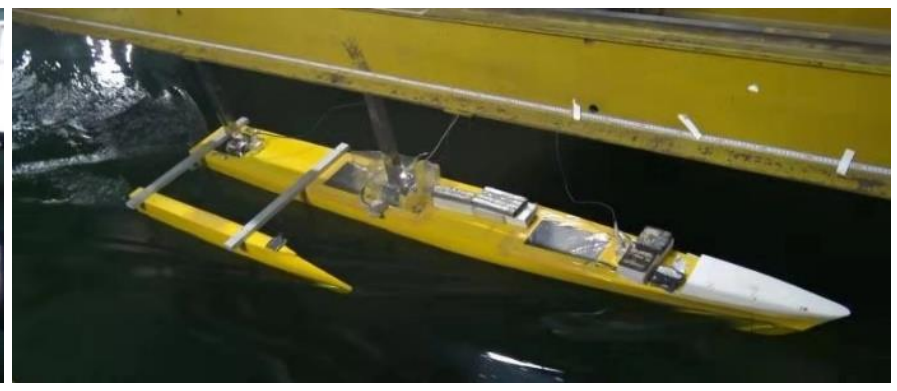

Fig. 17 Photograph of the experiment model

Fig.18 is the comparison of the experimental results of three longitudinal layouts, which are the curves of total drag coefficient, residual drag coefficient, sinkage and trim varying with Froude number. The experimental friction resistance is calculated by formulas (4), (10). The total resistance coefficient and residual resistance coefficient are calculated by formulas (11), (12). According to the analysis of the experimental results, the variation rules of the resistance and the trim with the longitudinal position of the side hull are basically consistent with the numerical results. 
Hu Jingfeng, Zhang Yihan,

Wang Ping, Qin Fei
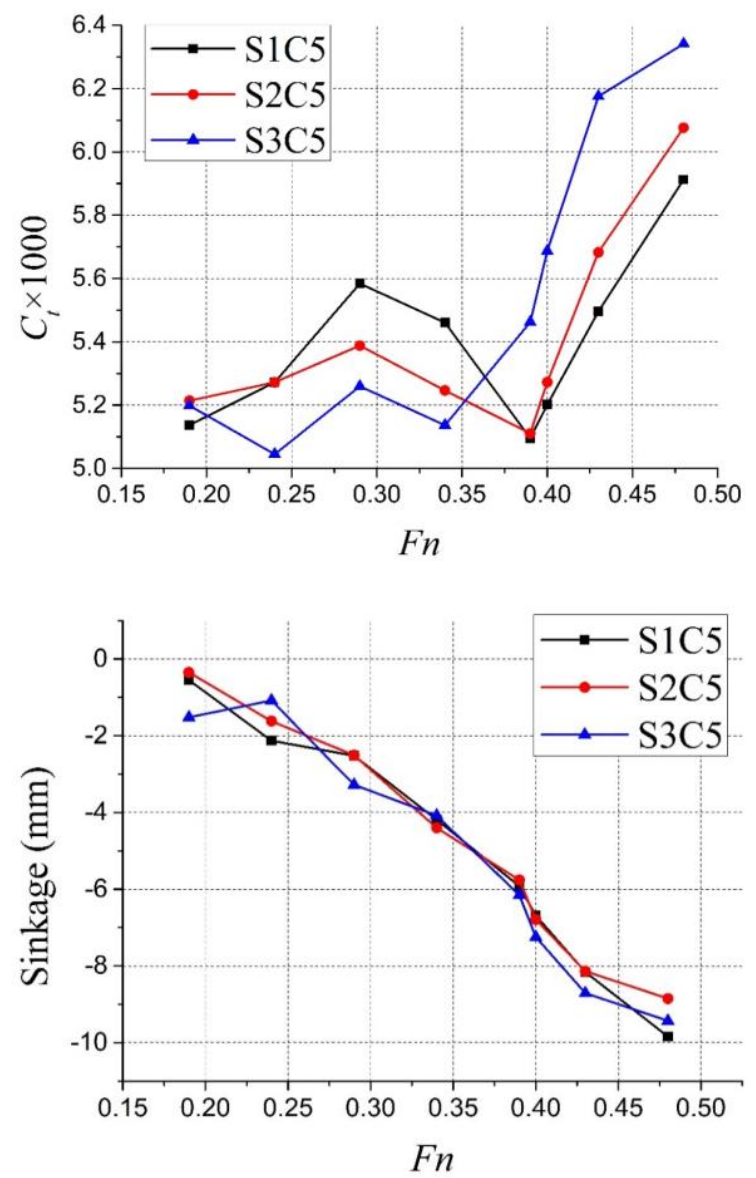

Numerical and Experimental Study on Resistance of Asymmetric Catamaran with Different Layouts
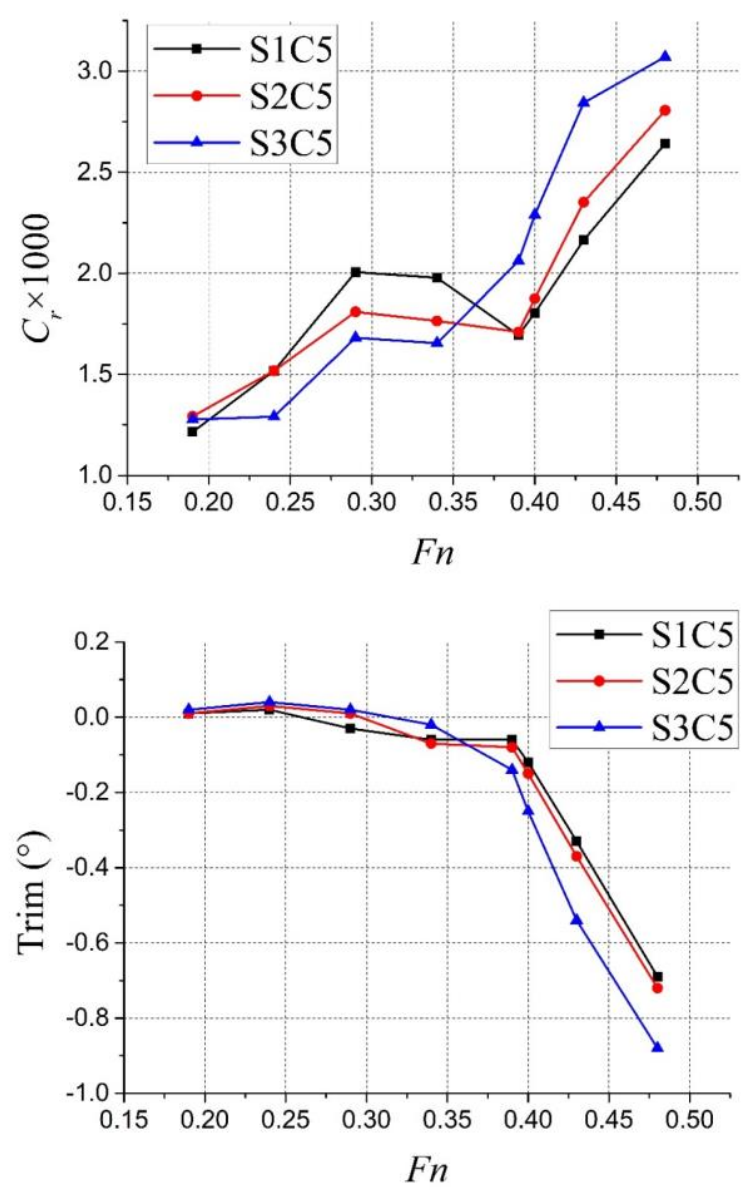

Fig. 18 Comparison of experimental results for different longitudinal layouts

The resistance, sinkage and trim of the models vary with Froude number are compared with the numerical ones, as shown in Fig.19-21. It can be seen that the CFD calculation results are in good agreement with the experimental values. However, the resistance and trim angle calculated by CFD are smaller than experiment at the high velocity $(F n=0.48)$.
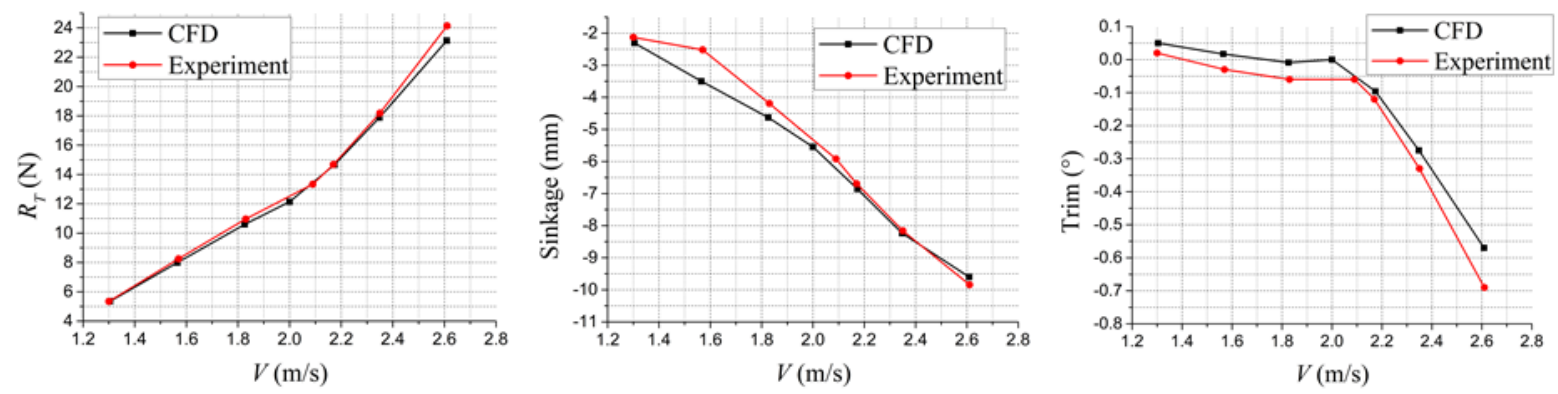

Fig. 19 Comparisons of numerical results and experimental results for layout S1C5
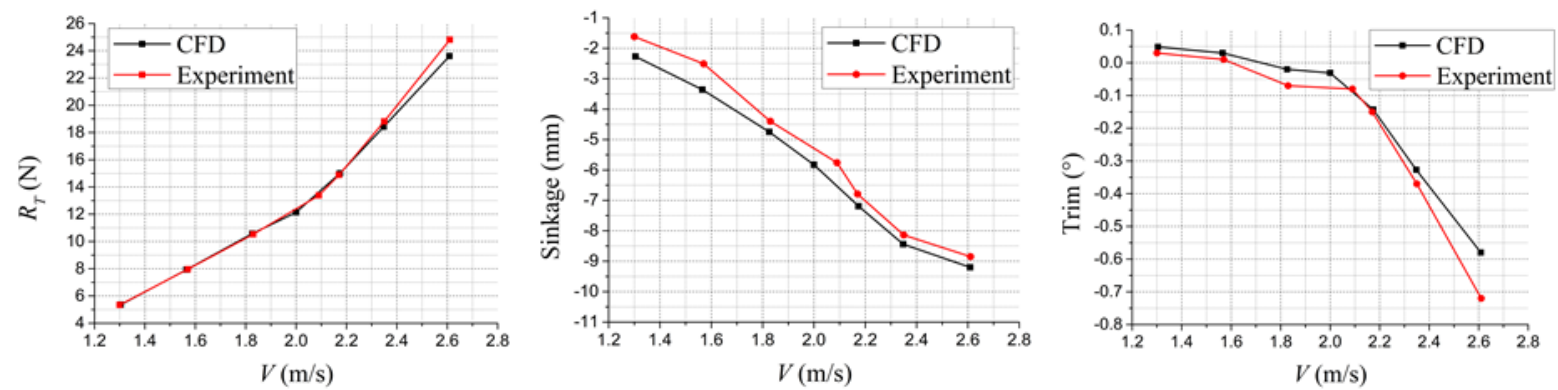

Fig. 20 Comparisons of numerical results and experimental results for layout S2C5 

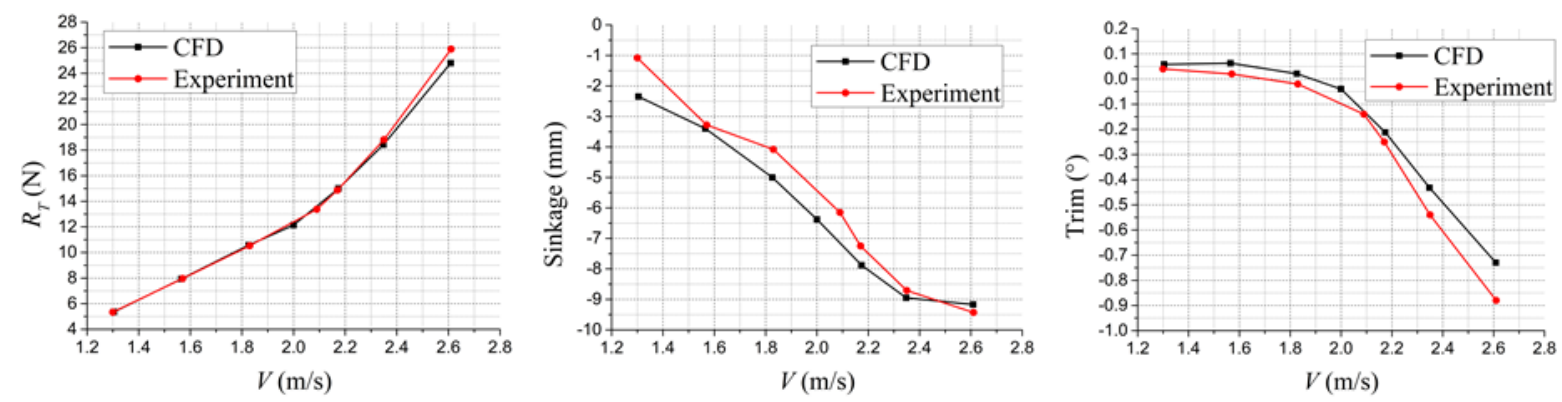

Fig. 21 Comparisons of numerical results and experimental results for layout S3C5

The relative difference of the total resistance values obtained for three configurations, $R_{T}^{C F D}$, from the experimental values, $R_{T}^{E X P}$, are given in Table 7 . The relative difference is calculated as follows:

$$
R D=\frac{R_{T}^{C F D}-R_{T}^{E X P}}{R_{T}^{E X P}} \cdot 100 \%
$$

It can be considered that the obtained numerical results show good agreement with experimental result. The largest relative difference for S1C5 equals $-4.18 \%$, for S2C5 $-4.84 \%$, for S3C5 5.42\%.

Table7. Total Resistance comparisons between CFD and experiment (Unit: N)

\begin{tabular}{|c|c|c|c|c|c|c|c|c|c|}
\hline \multirow{2}{*}{$F n$} & \multicolumn{3}{|c|}{ S1C5 } & \multicolumn{3}{|c|}{ S2C5 } & \multicolumn{3}{|c|}{ S3C5 } \\
\hline & $R_{T}^{C F D}$ & $R_{T}^{E X P}$ & $R D(\%)$ & $R_{T}^{C F D}$ & $R_{T}^{E X P}$ & $R D(\%)$ & $R_{T}^{C F D}$ & $R_{T}^{E X P}$ & $R D(\%)$ \\
\hline 0.24 & 5.35 & 5.34 & 0.22 & 5.36 & 5.34 & 0.37 & 5.39 & 5.11 & 5.42 \\
\hline 0.29 & 7.99 & 8.25 & -3.15 & 7.93 & 7.96 & -0.38 & 7.71 & 7.77 & -0.77 \\
\hline 0.34 & 10.60 & 10.96 & -3.33 & 10.57 & 10.53 & 0.38 & 10.44 & 10.31 & 1.21 \\
\hline 0.40 & 14.67 & 14.68 & -0.09 & 15.00 & 14.88 & 0.82 & 16.20 & 16.05 & 0.93 \\
\hline 0.43 & 17.88 & 18.19 & -1.70 & 18.42 & 18.81 & -2.07 & 19.85 & 20.44 & -2.89 \\
\hline 0.48 & 23.13 & 24.14 & -4.18 & 23.61 & 24.81 & -4.84 & 24.80 & 25.89 & -4.21 \\
\hline
\end{tabular}

\section{CONCLUSIONS AND OUTLOOK}

The simulations of the flow around the asymmetric catamaran which consider sinkage and trim have been carried out by means of the numerical solution of RANS equations. By the analysis of the numerical results, some characteristics of the resistance of different layouts have been obtained. The attention has also been devoted to the studies of the effect of the hull stagger distance on wave interference. The wave patterns, wave cuts have been gained to show a better insight into the wave field. The obtained numerical results show that, the resistances of the configuration with aft side hull longitudinal locations $(S L=0)$ do not range much with changing the lateral spacing from $0.8 B_{m}$ to $2.0 B_{m}$. While at $F n=0.40$, increasing the lateral spacing has a certain effect on reducing resistance when the lateral spacing is smaller than $1.4 B_{m}$, and the effect is less than $5 \%$. This effect is not obvious when the lateral spacing is greater than $1.4 B_{m}$.

The wave field is related with the resistance, trim and sinkage curve, as well as with the interference. At lower speed $(F n=0.29)$, the forward placing of side hull can effectively reduce the wave-making amplitude of the stern wave, which leads a smaller resistance. However, it has been shown that, at higher speed $(F n=0.40)$, the larger the stagger distance $(S L)$, the greater are the resistance, trim and sinkage, the wave cuts also show greater amplitude. The forward placing of side hull can cause the first peak of stern wave-making 
increase, and the interaction between main and side hull is obviously aggravated, also the bow of side hull locates at the peak position of wave behind the main hull. Consequently, the higher is the value for the resistance coefficient.

Moreover, experiments have been performed for three stagger distances at the Model towing tank of Harbin Engineering University. The numerical results of the resistance, sinkage and trim were validated against experimental data and satisfactory agreement was achieved. Both CFD and test results have shown that the resistance is sensitive to the longitudinal position of the side hull. The resistance reduction effect between the aft and forward side hull longitudinal locations can be $10 \%-14 \%$ at higher speed. Moreover, at lower speed $(F n=0.24 \sim 0.35)$, the resistance is smaller when the side hull is closer to the middle of the main hull; at higher speed $(F n=0.35 \sim 0.48)$, the resistance is smaller when the side hull is closer to the stern of the main hull.

\section{ACKNOWLEDGEMENTS}

This work was supported by the National Key Research and Development Program of China [2018YFC1406000]. It was also supported by the Ship Advance Research Project (30203020101). The authors would like to thank the College of Shipbuilding Engineering, Harbin Engineering University, for giving support to the experiment work.

\section{REFERENCES}

[1] Lian-en Zhao, Yong-he Xie. Principles and Design of High Performance Ships [M]. National Defense Industry Press, 2009.

[2] Mortaza Azizi Yengejeh, Mojtaba Maali Amiri, Hamid Mehdigholi,Mohammad Saeed Seif, Omar Yaakob. Numerical study on interference effects and wetted area pattern of asymmetric planing catamarans[J]. Proceedings of the Institution of Mechanical Engineers,2016,230(2). https://doi.org/10.1177/1475090215586638

[3] A. Hajiabadi, R. Shafaghat, H. Kazemi Moghadam. A study into the effect of loading conditions on the resistance of asymmetric high-speed catamaran based on experimental tests[J]. Alexandria Engineering Journal,2017. https://doi.org/10.1016/j.aej.2017.03.045

[4] O.B. Yaakob, A. Nasirudin,M.P. Abdul Ghani, T. Mat Lazim,M.A. Abd Mukti, Y.M. Ahmed. Parametric study of a low wake-wash inland waterways catamaran[J]. Scientia Iranica,2012,19(3). https://doi.org/10.1016/j.scient.2012.04.014

[5] S. Ikezoea, N. Hiratab, H. Yasukawab. Experimental Study on Seakeeping Performance of a Catamaran with Asymmetric Demi-Hulls. Jurnal Teknologi.66:2 (2014) 107-111 । https://doi.org/10.11113/jt.v66.2494

[6] Sang-Won Kim, Gyeong-Woo Lee and Kwang-Cheol Seo.The comparison on resistance performance and running attitude of asymmetric catamaran changing shape of tunnel stern exit region.IOP Conf. Series: Materials Science and Engineering 383 (2018) 012047. https://doi.org/10.1088/1757-899X/383/1/012047

[7] Guang-nian LI, Lian-en ZHAO, Qing-ren CHEN, Xiao-fei HU, Yonghe XIE, et al. Wave-Making Problem and Optimal Solution of Piece Hull Arrangement for Asymmetric Catamaran [J]. SHIPBUILDING OF CHINA, 2013(4):55-62.

[8] Guang-nian LI, Qing-ren CHEN, XIE Yong-he, Xiao-fei HU, et al. Computational research on wavemaking resistance of asymmetric catamaran based on the panel method in time domain[J]. Journal of Hydrodynamics, Ser.A,2013, 28(3).

[9] Dong-chi Yu,Pierre Lecointre,Ronald W. Yeung. Experimentally-based investigation of effects of wave interference on the wave resistance of asymmetric di-hulls[J]. Applied Ocean Research,2017,65. https://doi.org/10.1016/j.apor.2017.03.018

[10] Insel, M., Molland, A.F., 1992. An investigation into the resistance components of high speed displacement catamarans. R. Inst. Nav. Archit. 134, 1-20. 
[11] Broglia R , Zaghi S , Mascio A D . Numerical simulation of interference effects for a high-speed catamaran[J]. Journal of Marine Science \& Technology, 2011, 16(3):254-269. https://doi.org/10.1007/s00773-011-0132-3

[12] Zaghi S , Broglia R , Mascio A D . Analysis of the interference effects for high-speed catamarans by model tests and numerical simulations[J]. ocean engineering, 2011, 38(17-18):2110-2122. https://doi.org/10.1016/j.oceaneng.2011.09.037

[13] Broglia R , Jacob B , Zaghi S , et al. Experimental investigation of interference effects for high-speed catamarans[J]. Ocean $\quad$ Engineering, $\quad$ 2014, 76(jan.15):75-85. https://doi.org/10.1016/j.oceaneng.2013.12.003

[14] Farkas A, Degiuli N , Martic I . Numerical investigation into the interaction of resistance components for a series 60 catamaran[J]. Ocean Engineering, 2017, 146(dec.1):151-169. https://doi.org/10.1016/j.oceaneng.2017.09.043

[15] He, W., Castiglione, T., Kandasamy, M., Stern, F., 2015. Numerical analysis of the interference effects on resistance, sinkage and trim of a fast catamaran. J. Mar. Sci. Technol. 20 (2), 292-308. https://doi.org/10.1007/s00773-014-0283-0

[16] Xin-gong C , Ping W , Xiao-min X . Resistance study on alterative layouts of the trimaran hull configuration[J]. journal of hydrodynamics(ser.a), 2007.

[17] Ping W, Xin-gong C, Yun H, et al. Experimental and theoretical study on hydrodynamic arrangement law of trimaran lateral position [C]// Ship Mechanics Academic Conference and 10th Anniversary of Ship Mechanics Founding. 2007.

[18] Mizine, I., Karafiath, G., Queutey, P. and Visonneau, M. (2009), "Interference Phenomenon in Design of Trimaran Ship", Proceedings of the 10th International Conference on Fast Sea Transportation - FAST 2009, Athens, Greece, October 2009

[19] Duy, T.N., Hino, T. and Kazuo, S.: Numerical study on stern flow fields of ship hulls with different transom configurations [J]. Ocean Engineering, Vol. 129, 2017, pp. 401-414. https://doi.org/10.1016/j.oceaneng.2016.10.052

[20] Muscari, R., Dubbioso, G., Viviani, M., and Mascio, A.D.: Analysis of the asymmetric behavior of propeller-rudder system of twin screw ships by CFD [J]. Ocean Engineering, Vol. 129, 2017, pp. 269281. https://doi.org/10.1016/j.oceaneng.2017.07.056

[21] Ozdemir, Y.H., Cosgun, T., Dogrul, A., and Barlas, B. A Numerical application to predict the resistance and wave pattern of Kriso Container Ship [J], Brodogradnja, Vol. 67, No:2, 2016, pp. 47-65. https://doi.org/10.21278/brod67204

[22] Farkas, A., Degiuli, N., and Martic, I. Numerical simulation of the viscous flow around a tanker model [J], Brodogradnja, Vol. 68, No:2, 2017, pp. 109-125. https://doi.org/10.21278/brod68208

[23] Ozdemir, Y.H., Barlas, B. Free surface flow simulation around an appended ship hull [J]. Brodogradnja, Vol. 69, No:3, 2018, pp. 25-41. https://doi.org/10.21278/brod69302

[24] Zhong WANG,Xiao-ping LU. Numerical simulation of wave resistance of trimarans by nonlinear wave making theory with sinking and trim being taken into account $[\mathrm{J}]$. Journal of Hydrodynamics, Ser.B,2011,23(2). https://doi.org/10.1016/S1001-6058(10)60107-2

[25] Choi, Junwoo and Sung, Bum Yoon. 2009. "Numerical simulations using momentum source wave-maker applied to RANS equation model”, Coastal Engineering, 56 (10), pp. $1043 \quad$ - 1060. https://doi.org/10.1016/j.coastaleng.2009.06.009

[26] ZHAO Fa-ming, GAO Cheng-jun, XIA Qiong, et al. Overset mesh research on the application of ship CFD[J]. Journal of Ship Mechanics, 2011, 15(4):332-341.

[27] Mancini S, Begovic E, Day A.H, Incecik A, et al. Verification and validation of numerical modelling of DTMB 5415 roll decay[J]. Ocean Engineering, 2018, 162(aug.15):209-223. https://doi.org/10.1016/j.oceaneng.2018.05.031

[28] ITTC-Recommended Procedures and Guidelines (2014) "Practical Guidelines for ship CFD Applications"

[29] Rui DENG, De-bo HUANG, Guang-li ZHOU, Hua-wei SUN, et al. Investigation on Some Factors Effecting Ship Resistance Calculation with CFD Code FLUENT [J]. Journal of Ship Mechanics, 2013(6):616-624.

[30] ITTC-Recommended Procedures and Guidelines (2002) "CFD General Uncertainty Analysis and Validation Methodology and Procedures" 
Hu Jingfeng, Zhang Yihan, Wang Ping, Qin Fei
Numerical and Experimental Study on Resistance of Asymmetric Catamaran with Different Layouts

[31] Yengejeh, M.A., Amiri, M.M., Mehdigholi, H., Seif, M.S., Yaakob, O., 2016. Numerical study on interference effects and wetted area pattern of asymmetric planing catamarans. Proc. Institute. Mech. Eng. Part M J. Eng. Marit. Environ. 230 (2), 417-433. https://doi.org/10.1177/1475090215586638

[32] G-L Zhou, D-B Huang, F-L Li, et al. Uncertainty analysis of ship model towing resistance test [J]. Journal of Harbin Engineering University, 2006 (03): 377-381 + 390

Submitted: $\quad$ 25.10.2019. Jingfeng Hu, huakej1992@126.com,

Marine Design \& Research Institute of China, No.1688 South Xi Zang Road, Shanghai, China.

Yihan Zhang, zhangyihan437@163.com,

Marine Design \& Research Institute of China, No.1688 South Xi Zang Road, Shanghai, China.

Ping Wang, hongliu@shmtu.edn.cn

Marine Design \& Research Institute of China, No.1688 South Xi Zang Road, Shanghai, China.

Fei Qin, 18801903337@139.com

Marine Design \& Research Institute of China, No.1688 South Xi Zang Road, Shanghai, China. 